\title{
A comprehensive overview of metaplastic breast cancer: clinical features and molecular aberrations
}

Tejaswini P. Reddy ${ }^{1,2}$, Roberto R. Rosato ${ }^{1}$, Xiaoxian Li ${ }^{3}$, Stacy Moulder ${ }^{4}$, Helen Piwnica-Worms ${ }^{4}$ and Jenny C. Chang ${ }^{1,5^{*}}$

\begin{abstract}
Metaplastic breast cancer (MpBC) is an exceedingly rare breast cancer variant that is therapeutically challenging and aggressive. $\mathrm{MpBC}$ is defined by the histological presence of at least two cellular types, typically epithelial and mesenchymal components. This variant harbors a triple-negative breast cancer (TNBC) phenotype, yet has a worse prognosis and decreased survival compared to TNBC. There are currently no standardized treatment guidelines specifically for MpBC. However, prior studies have found that MpBC typically has molecular alterations in epithelialto-mesenchymal transition, amplification of epidermal growth factor receptor, PI3K/Akt signaling, nitric oxide signaling, Wnt/ $\beta$-catenin signaling, altered immune response, and cell cycle dysregulation. Some of these molecular alterations have been studied as therapeutic targets, in both the preclinical and clinical setting. This current review discusses the histological organization and cellular origins of $\mathrm{MpBC}$, molecular alterations, the role of radiation therapy, and current clinical trials for MpBC.
\end{abstract}

Keywords: Metaplastic breast cancer, PI3K signaling, NOS signaling, Epithelial-to-mesenchymal transition

\section{Introduction}

Metaplastic breast cancer $(\mathrm{MpBC})$ is a rare and aggressive malignancy that accounts for $0.2-5 \%$ of all breast cancers, and as such, $\mathrm{MpBC}$ carries the worst prognosis in comparison to other breast cancer types and plays a significant role in global breast cancer mortality [1]. In the SEER database from 1973 to 2015, there were less than 10,000 cases of MpBC in the USA annually [2]. This malignancy is characterized by the histological presence of at least two cellular types, typically epithelial and mesenchymal components [3]. $\mathrm{MpBC}$ is typically a triple-negative breast cancer (TNBC), meaning the

\footnotetext{
* Correspondence: jcchang@houstonmethodist.org

'Houston Methodist Research Institute, 6670 Bertner Ave, Houston, TX 77030, USA

${ }^{5}$ Houston Methodist Cancer Center/Weill Cornell Medicine, OPC 24, 6445 Main Street, Houston, TX 77030, USA

Full list of author information is available at the end of the article
}

tumor lacks the expression of estrogen receptor (ER), progesterone receptor (PR), and human epidermal growth factor 2 receptor (HER2) [3]. Unfortunately, $\mathrm{MpBC}$ carries a worse prognosis in comparison to nonmetaplastic TNBC, has twice the risk of recurrence, and has a shorter disease-free and overall survival (OS) [4]. The term "metaplastic carcinoma" was first published by Huvos and colleagues in 1973 [5]. Due to its rare and aggressive nature, there have been many limitations in completely delineating the molecular and genetic landscape of $\mathrm{MpBC}$. However, an emerging number of $\mathrm{MpBC}$ case studies, enhanced technology in research, and increased awareness of this rare breast neoplasm have allowed clinicians and researchers to gain a better understanding of the morphology, prognosis, molecular alterations, and potential treatment options. The goal of this review is to provide an overview of $\mathrm{MpBC}$, including 
cellular and molecular characteristics, histopathology, treatment options, and ongoing clinical trials.

\section{Histological organization of MpBC}

By definition, metaplastic carcinomas contain one or more cell populations that have undergone metaplastic differentiation, meaning that cells have converted from glandular to non-glandular morphology [6]. These metaplastic changes include carcinomatous (squamous) and sarcomatous elements, including osseous, chondroid, and spindle morphology [1]. The WHO Classification of Breast Tumors classifies $\mathrm{MpBC}$ as mixed metaplastic carcinoma, low-grade adenosquamous carcinoma, fibromatosis-like, squamous cell carcinoma, spindle cell carcinoma, and metaplastic carcinoma with mesenchymal differentiation $[1,7]$. All of these metaplastic variants are aggressive and chemoresistant and have a high propensity to metastasize, except fibromatosis-like carcinoma and low-grade adenosquamous carcinoma [8].

Understanding the differences between histological variants may provide insight into clinical prognosis and potential therapeutic options. For example, Schwartz and colleagues organized $\mathrm{MpBC}$ into epithelial and mixed types, in which epithelial types included squamous cell carcinoma, adenocarcinoma with spindle cell differentiation, and adenosquamous carcinoma, whereas mixed types included carcinoma with chondroid metaplasia, carcinoma with osseous metaplasia, and carcinosarcoma [9]. Tse and colleagues classified MpBC into three groups, epithelial-only carcinoma, biphasic epithelial and sarcomatoid carcinoma, and monophasic spindle cell carcinoma [10]. Oberman conducted a clinicopathological study of 29 patients with primary breast neoplasms and classified MpBC into spindle cell carcinoma, invasive ductal carcinoma with extensive squamous metaplasia, and invasive ductal carcinoma with pseudosarcomatous metaplasia [11]. One of the primary issues with these descriptive classifications is the lack of correlation between the microscopic pattern and prognosis of the disease, in part due to the rarity of the disease.

Of these, one publication by Song et al. found that there was clinical significance to sub-classifying $\mathrm{MpBC}$ [12]. This study showed that the prognosis of $\mathrm{MpBC}$ was worse than triple-negative invasive ductal carcinoma (TN-IDC), with a 5 -year overall survival rate of $54.5 \%$ vs. $73.3 \%$, respectively. Adenocarcinoma with spindle cell differentiation had the worst 5-year overall survival rate at $40 \%$. Overall, this study concluded that separating MpBC based on histological variants may have clinical significance.

The prognosis and response to therapy vary among the metaplastic variants. $\mathrm{MpBC}$ also shows increased locoregional and distant tumor recurrence and is far more aggressive than invasive ductal carcinoma, even when matched for age, stage, and tumor grade [13]. In addition, the majority of $\mathrm{MpBC}$ is triple-negative, yet the genomic, transcriptomic, and proteomic characteristics between MpBC and TNBC differ. Examples of this include the marker p63, which is commonly expressed in spindle cell carcinoma, or that low-grade adenosquamous carcinoma harbor high rates of PIK3CA mutations, but no TP53 mutations and androgen receptor expression. Conventional TNBC has a low rate of PIK3CA mutations [14, 15].

A genomics profiling study performed by McQuerry and colleagues found that MpBC samples with mesenchymal (osteoid or chondroid) histology had increased Snail, BCL-2-like-1 protein, and Akt1 pathway activity in comparison to non-mesenchymal MpBC tumors [16]. When comparing the gene expression profiles of $\mathrm{MpBC}$ to TNBC tumors, MpBC tumors had more upregulation of epithelial-to-mesenchymal transition (EMT) and collagen genes, but downregulation of late cornified envelope and keratinization genes. Overall, these results support that $\mathrm{MpBC}$ histological variants may exhibit different genomic profiles and that EMT may play an influential role in the aggressiveness and lethality of this rare breast cancer subset.

A proteomics study by Djomehri and colleagues discovered potential metaplastic pathological subtype-specific biomarkers/therapeutic targets as well as proteomic differences between $\mathrm{MpBC}$ and TNBC [17]. In this study, they performed multiplex quantitative tandem mass tag-based proteomics and gene set enrichment analysis to elucidate unique protein signatures in TNBC, MpBC pathological variants (spindle, squamous, and sarcomatoid $\mathrm{MpBC}$ ), and normal mammary tissue. Compared to the TNBC proteome, the top upregulated pathway in the $\mathrm{MpBC}$ proteome was EMT and the top downregulated pathway was oxidative phosphorylation (OXPHOS). When proteomes of the specific $\mathrm{MpBC}$ pathological subtypes were compared, they discovered that spindle $\mathrm{MpBC}$ was highly enriched with MYC and E2F targets and ribosomal pathway proteins. Squamous MpBC had elevated interferon-gamma signaling/broad inflammatory responses, TP53 and PI3K signaling, apical junction signaling, and decreased OXPHOS, $\mathrm{MYC}$, and E2F targets. Sarcomatoid MpBC displayed high EMT and OXPHOS signaling and low PI3K/MTORC1 and interferon-gamma signaling. The discovery of unique proteomic features among the $\mathrm{MpBC}$ pathological variants may provide new insight into novel treatment strategies to improve the survival of $\mathrm{MpBC}$ patients. Nevertheless, more studies and discussions are needed to examine the clinical value of separating $\mathrm{MpBC}$ based on the histological variant.

\section{Cell origin of $\mathrm{MpBC}$}

The clonality and origin of $\mathrm{MpBC}$ have been debated for years, and there are at least three hypotheses to explain 
why $\mathrm{MpBC}$ are biphasic tumors, meaning the presence of sarcomatous and carcinomatous components within the same tumor. The collision theory suggests that carcinomatous and sarcomatous tissues are derived from separate progenitor cells [9], while the combination theory of monoclonal origin suggests that a common multipotent progenitor cell is responsible for giving rise to both sarcomatous and carcinomatous cells. The conversion/metaplastic theory suggests that the sarcomatous components derive from the carcinomatous component via a metaplastic process. Evidence to support the metaplastic theory comes from data that showed both epithelial and mesenchymal components of the tumor display a positive expression of cytokeratin, S-100, and vimentin [18]. Furthermore, it has also been suggested that MpBC may derive from myoepithelial cells, as the tumors are frequently positive for myoepithelial markers including CD10, p63, and smooth muscle actin [19].

\section{Molecular alterations in $\mathrm{MpBC}$}

Despite its rarity, there have been some studies that have revealed molecular alterations and actionable genetic changes within $\mathrm{MpBC}$. Molecular analysis of $\mathrm{MpBC}$ will be crucial to identify potential options for targeted therapeutic intervention and for promising clinical strategies. Here, we provide an overview of molecular alterations and characteristics found in $\mathrm{MpBC}$ cases, which is also summarized in Table 1.

\section{Epithelial-to-mesenchymal transition}

Epithelial-to-mesenchymal transition (EMT) is a transient process in which epithelial cells lose their cell polarity and cell-cell adhesion qualities and acquire mesenchymal properties, including enhanced migratory capacity, resistance to apoptosis and chemotherapy, invasiveness, and characteristic morphological and gene expression changes [38]. EMT after embryogenesis is considered pathological, and this process is associated with a loss of E-cadherin and claudin expression and enhanced expression of mesenchymal markers such as vimentin and smooth muscle cell actin [39]. EMT is typically regulated by transcription factors (TF) such as Goosecoid, Snail, Slug, Twist, FOXC1, FOXC2, Zeb1, and Zeb2 [20]. While EMT has been extensively studied in claudin-low and metaplastic breast cancers, EMT has also been involved in tumorigenesis of other cancers, such as liver, lung, prostate, pancreas, thyroid, and glioblastoma multiforme [40, 41]. In general, tumors that undergo EMT typically harbor an epithelial phenotype and have activated EMT-TF-dependent cellular processes, such as dedifferentiation and plasticity [41]. Taube and colleagues conducted a study in which they identified an EMT core genetic signature that was enriched for genes regulated by Zeb1 and this genetic signature was similar to that of claudin-low and metaplastic breast cancers [20]. Hennessy et al. found comparable results in their study, in which $28 \mathrm{MpBC}$ tumor samples were transcriptionally profiled and probed using a tumor-initiating cell (TIC) gene signature [21]. This TIC gene signature was developed by Creighton et al. [22]. MpBC tumors shared a similar genetic signature to TIC and claudin-low breast cancer gene signatures, had more stem-cell-like features, and expressed high levels of EMT markers [21]. TICs are more prominently observed following endocrine therapy or chemotherapy, have intrinsic resistance to chemotherapy, exhibit EMT characteristics, and are capable of self-renewal [22]. A crucial finding from this study was that MpBC showed a high frequency of amplification, mutation, and activation of phosphoinositide 3-kinase (PI3K) signaling relative to basal and claudin-low breast cancers. Therefore, EMT/ stem-cell-like features in combination with PI3K signaling hyperactivation may provide an explanation for why $\mathrm{MpBC}$ is an aggressive, chemorefractory subtype and potentially originated from a chemoresistant stem cell.

\section{Epidermal growth factor receptor (EGFR)}

MpBC consistently overexpresses EGFR, but usually lacks HER2 overexpression and amplification [23]. ReisFilho and colleagues found that $34 \%$ of MpBC cases exhibit EGFR gene amplification and this gene amplification is associated with gene overexpression [24]. This study found no activating mutations in EGFR, suggesting that point mutations within the receptor are unlikely to influence the overexpression of EGFR. Another study assessed $77 \mathrm{MpBC}$ samples and found that the majority of the samples were positive for p63 (59\%), cytokeratin 5/6 (58\%), EGFR overexpression (66\%), and KIT (24\%) [25]. This study also found no activating mutations in EGFR and KIT. Fluorescence in situ hybridization was performed to show high EGFR copy number secondary to aneusomy (22\%) and amplification (4\%). To compare the differences in EGFR amplification among $\mathrm{MpBC}$ and mesenchymal and basal TNBC tumors, we performed droplet digital PCR analysis (ddPCR) to assess EGFR DNA copy number values. We used DNA isolated from well-established MpBC and TNBC patient-derived xenograft (PDX) tumors. The details of the experiment are included in the supplementary information. We found that in comparison to mesenchymal TNBC and MpBC, basal TNBC had the highest EGFR copy number values (Fig. 1). PDX BCM-4013 (basal-like 2 subtype, BL2) exhibited the most EGFR amplification among all PDXs. BL2 TNBC tumors have been shown to exhibit enhanced EGFR gene expression [42]. Fifty percent of the MpBC PDX tumors exhibited EGFR copy number values greater than two. These findings shed light on the differential expression of EGFR between TNBC and MpBC as 
Table 1 List of molecular alterations in MpBC

\begin{tabular}{ll}
\hline Molecular alteration & Description \\
\hline Epithelial-to-mesenchymal transition (EMT) & -EMT core genetic signature shares similarity to core genetic \\
& signatures of claudin-low and metaplastic breast cancers [20]. \\
& - MpBC tumors have more stem-like features, express high \\
& levels of EMT markers, and share a similar genetic signature to \\
& tumor-initiating cell (TIC) genetic signature [21]. \\
& - TICs are prominent following endocrine/chemotherapy, more \\
& chemoresistant, exhibit EMT, and can undergo self-renewal [22]. \\
& -34\% of MpBC cases exhibit EGFR gene amplification associated \\
& with gene overexpression and no EGFR activating mutations [23, 24]. \\
& -Fluorescent in situ hybridization showed high EGFR copy \\
Epidermal growth factor receptor & number secondary to aneusomy (22\%) and amplification (4\%) [25]. \\
(EGFR) signaling pathway & - Majority of MpBC is positive for p63 (59\%), cytokeratin 5/6 (58\%), \\
& KIT (24\%), and EGFR (66\%) overexpression [25].
\end{tabular}

Phosphoinositide 3-kinase (PI3K) signaling pathway

Nitric oxide synthase (NOS) signaling pathway

Wnt/ß-catenin signaling

Programmed cell death protein 1 (PD-1)/ programmed death ligand-1 (PD-L1)

Cell cycle regulation
$-47 \%$ of MpBC tumors harbor PIK3CA mutations and 5\% have PTEN deletions [21]. 36.4\% of HR+ breast cancers have PIK3CA mutations [26]. -Whole-exome sequencing analysis of $\mathrm{MpBC}$ tumors showed the most altered genes were PIK3CA (29\%), PIK3R1 (11\%), FAT1 (11\%), ARIDIA (11\%), and PTEN (11\%) [27].

-Next-generation sequencing of $\mathrm{MpBC}$ tumors showed the most commonly altered genes were TP53 (68.4\%), PIK3CA (42.1\%), and PTEN (15.8\%) [28].

-TNBC expresses high levels of nitric oxide (NO) than HER2+ or luminal breast cancers and enhanced inducible nitric oxide synthase (iNOS) expression is associated with worse prognosis and may confer resistance to chemotherapy [29].

-Inhibition of iNOS via L-NMMA in combination with docetaxel is more effective than docetaxel alone in enhancing tumor apoptosis, cell proliferation/migration, and reducing tumorinitiating capacity in TNBC and $\mathrm{MpBC}$ models.

-39/40 (97.5\%) of MpBC tumors harbor a RPL39 A14V oncogenic mutation, which is associated with enhanced NO activity, cancer cell stemness, and lung metastasis [30].

-Immunohistochemistry $(\mathrm{IHC})$ of $\mathrm{MpBC}$ samples showed aberrant $\beta$ catenin expression in 33/36 (92\% of cases), and mutational analysis showed that $25.9 \%$ of MpBC tumors had CTNNB1 missense mutations, $7.4 \%$ tumors had APC mutations, and $18.5 \%$ tumors had WISP3 mutations [31].

$-\mathrm{HC}$ of $\mathrm{MpBC}$ tumor samples reveals that $\beta$-catenin expression has more focal nuclear localization [32].

$-\mathrm{MpBC}$ tumors commonly harbor mutations in $\mathrm{Wnt} / \beta$-catenin signaling and PI3K/Akt signaling than TNBC tumors [27]. -The levels of CCN6 are low in MpBC, leading to enhanced insulin-like growth factor 1 levels, EMT, invasion, metastasis, and bone morphogenic-4 signaling [8].

-A mouse model of mammary epithelium-specific $\mathrm{C}(n 6$ protein deletion (MMTV-cre; $\left(\mathrm{Cn} 6^{\mathrm{fl} / \mathrm{f}}\right.$ ) has been developed, which can recapitulate many features of human spindle MpBC tumors [17, 33].

-PD-L1 is expressed more in MpBC tumors (46\%) relative to other breast tumor types (6\% in HR+ and 9\% in HER2+ breast cancers [34]. -Another study performed PD-L1 immunohistochemical staining of 21 MpBC tumor samples and found that PD-L1 expression was associated with a worse RFS and OS [28].

-A patient with metastatic MpBC (PD-L1+ and with PIK3CA

H1047L mutation) showed a dramatic response to pembrolizumab in combination with nab-paclitaxel [35].

-MpBC tumors harbor a high frequency of TP53 (64\%) and TERT (catalytic subunit of telomerase) promoter mutations (25\%) [36] -TERT mutations are commonly found in the spindle and squamous MpBC [36]

-Myoepithelial MpBC shows a 9p21.3 chromosomal loss, including loss of genes CDK2NA and CDK2NB, which code for cyclin-dependent kinase inhibitors p16 $6^{\text {INK4a }}$ and p15 $5^{\text {INK4b }}$ [37].

$-64.3 \%$ of myoepithelial MpBC tumors with 9p21.3 loss also had a PIK3CA mutation [37].

\begin{tabular}{ll} 
Reference & $\begin{array}{l}\text { Sample } \\
\text { size }\end{array}$ \\
\hline Taube et al. [20] & 244 \\
Hennessy et al. [21] & 28
\end{tabular}

Reis-Filho et al. [23] 25

Reis-Filho et al. [24] 47

Gilbert et al. [25] $\quad 38$

Hennessy et al. [21] 28

Razavi et al. [26] 1918

Ng et al. [27] $\quad 35$

Afkhami et al. [28] 21

Granados-Principal

et al. [29]

Dave et al. [30]

Hayes et al. [31]

Lacroix-Triki et al. [32] 52

$\mathrm{Ng}$ et al. [27] $\quad 35$

Martin et al. [33] _

Joneja et al. [34] 290

Afkhami et al. [28] 21

Adams 2017 [35] 1

Krings and Chen [36] 28

Bartels et al. [37] 34 


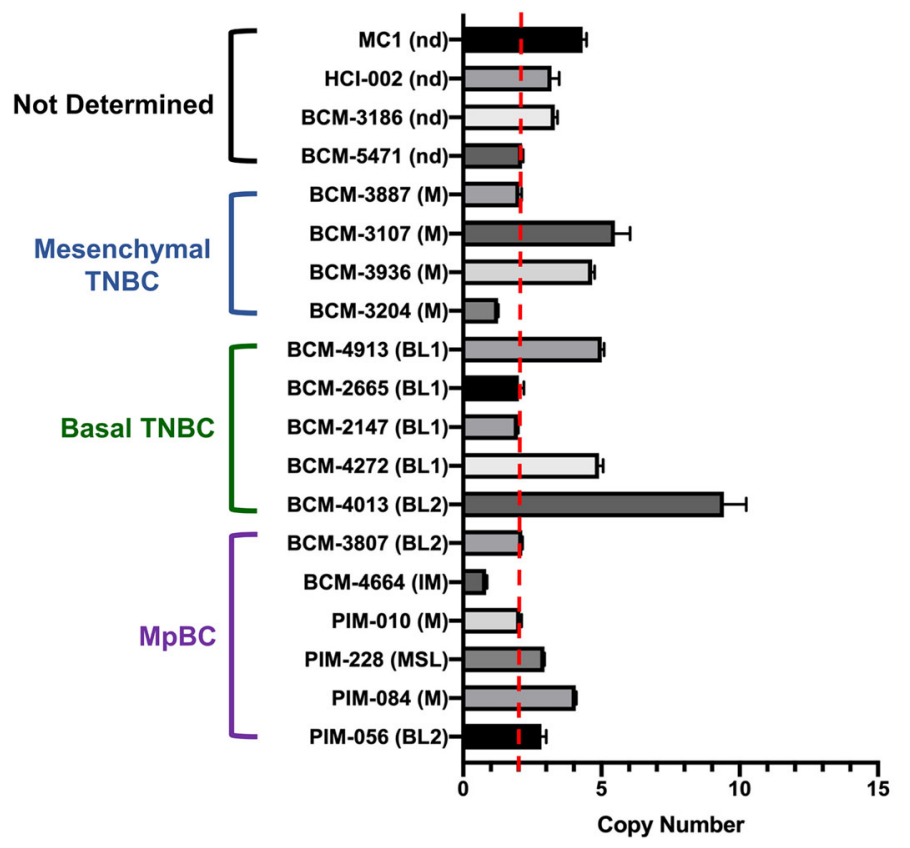

Fig. 1 EGFR copy number variant values of mesenchymal TNBC, basal TNBC, and MpBC PDX tumors. Droplet digital PCR was performed using DNA isolated from PDX tumors, and EGFR and RPP30 (reference gene)-specific primers and probes. Red dotted line indicates the normal copy number threshold (CN 2). Pietenpol Classification [42]: BL1, basal-like 1; BL2, basal-like 2; IM, immunomodulatory; LAR, luminal androgen receptor; M, mesenchymal; MSL, mesenchymal-stem like; nd, not determined

well as warrant further investigation on using EGFR tyrosine kinase inhibitors as therapeutics against MpBC.

\section{Phosphoinositide 3-kinase (PI3K) pathway}

The PI3K/Akt/mTOR signaling pathway controls cellular growth, proliferation, metabolism, cellular survival, and angiogenesis and is one of the most frequently dysregulated pathways in cancer [43]. The pathway can be hyperactivated in cells through diverse genomic alterations, such as mutations in PIK3CA, PIK3R1, AKT1, $M T O R$, TSC2, and $L K B 1$, as well as various other tumor suppressor genes and oncogenes [44]. PIK3CA codes for $\mathrm{p} 100 \alpha$, the catalytic subunit of the PI3K $\alpha$ complex, and phosphatase and tensin homolog (PTEN) is a tumor suppressor gene of this pathway that is frequently deleted in cancers. A seminal study in the field of $\mathrm{MpBC}$ research found that this rare cancer type harbors 47\% PIK3CA mutations and $\sim 5 \%$ PTEN deletions [21]. MpBC tumors harbor more PIK3CA mutations than hormone receptor $(\mathrm{HR})+$ breast cancers, in which $36.4 \%$ of those cancers have a PIK3CA mutation [26]. However, the increased percentage of $\mathrm{MpBC}$ with PIK3CA mutations may be a biased result, as this study had a small sample size of MpBC tumors. Nevertheless, another study conducted whole-exome sequencing on $\mathrm{MpBC}$ samples and found that $\mathrm{MpBC}$ tissue contained genetic alterations in PIK3CA (29\%), PIK3R1 (11\%), FAT1 (11\%), ARID1A
(11\%), and PTEN (11\%) [27]. A next-generation sequencing mutational assay on tumor samples from $19 \mathrm{MpBC}$ patients found comparable results and showed that the most commonly altered genes in MpBC were TP53 (68.4\%, 13/19), PIK3CA (42.1\%, 8/19), and PTEN (15.8\%, 3/19) [28]. The study also showed that recurrence-free survival (RFS) and OS were significantly worse for MpBC patients with PIK3CA mutations. We analyzed a publicly available cancer cohort $(n=9052)$ from cBioPortal to identify alteration frequencies for $E G F R$, PIK3CA, and PTEN genes across all breast cancer subtypes (Fig. 2). Across all cancer types, $\mathrm{MpBC}$ showed the highest frequency of alterations in EGFR and PTEN, and a modest percentage of PIK3CA mutations. While it is difficult to conclude whether PIK3CA mutations are more commonly seen in $\mathrm{MpBC}$ vs. $\mathrm{TNBC}$, largely due to the rare nature of $\mathrm{MpBC}$, we suggest that targeting this pathway with isoform-specific inhibitors in combination with other novel therapeutics may be the future for $\mathrm{MpBC}$ treatment. From the same database, we also compared the OS of $33 \mathrm{MpBC}$ patients to 7515 non-metaplastic breast cancer patients (including HR+, HER2+, and TNBC) and found MpBC had a poorer OS (HR 11.5, 95\% CI 3.6436.35) than non-metaplastic breast cancer (Fig. 3). The median survival rate for $\mathrm{MpBC}$ patients was 64.4 months, versus 159.2 months for patients diagnosed with nonmetaplastic breast cancers. These findings further support 


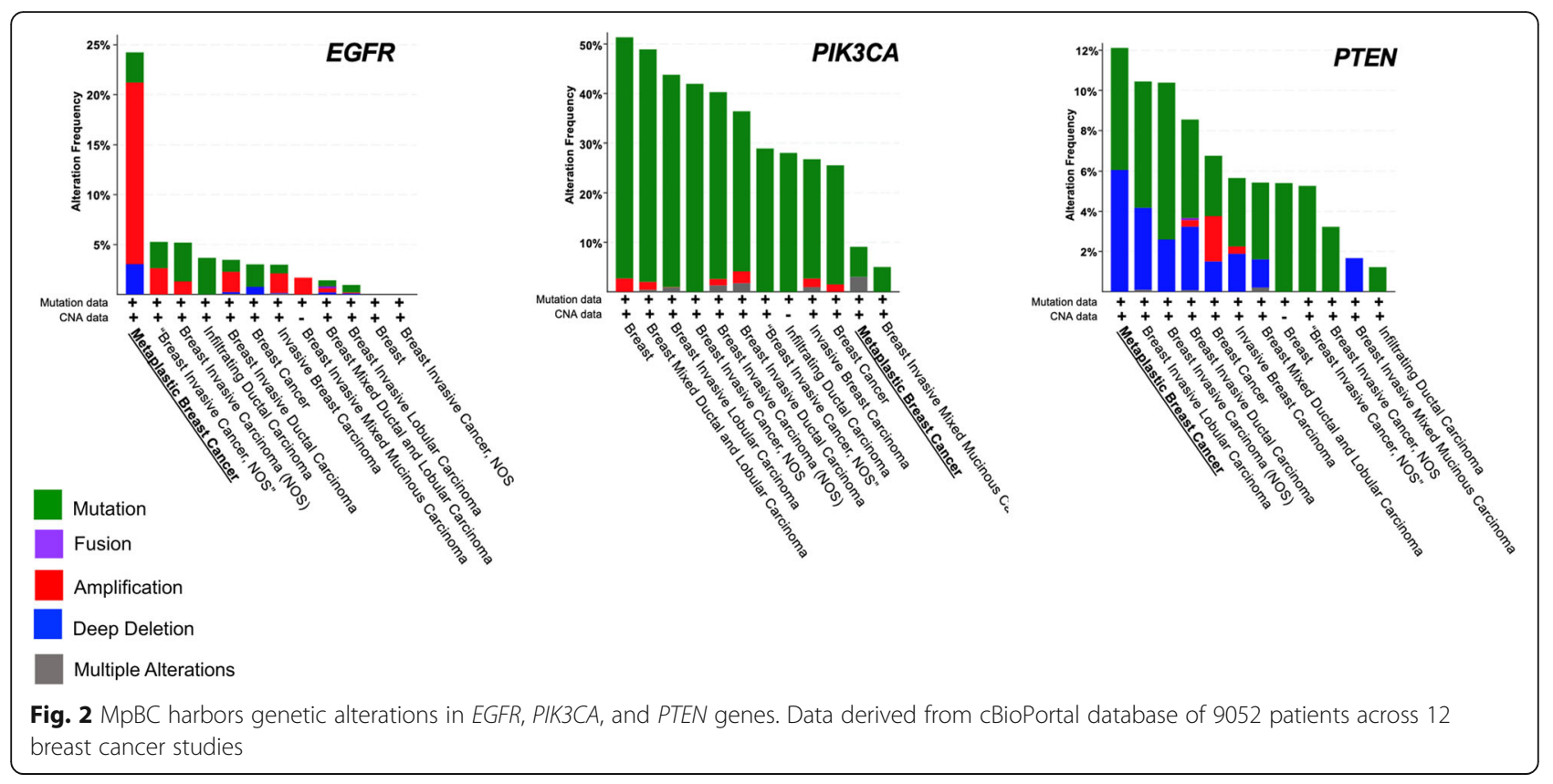

studies that have described $\mathrm{MpBC}$ as highly aggressive cancer with poorer clinical outcomes than other breast malignancies $[4,45,46]$.

\section{Nitric oxide signaling pathway}

TNBC expresses higher levels of nitric oxide (NO), which is estimated by nitrate levels, than HER2+ or luminal breast cancers [47]. Furthermore, previous in vitro studies have shown that TNBC tumors expressing inducible nitric oxide synthase (iNOS) produce moderate to

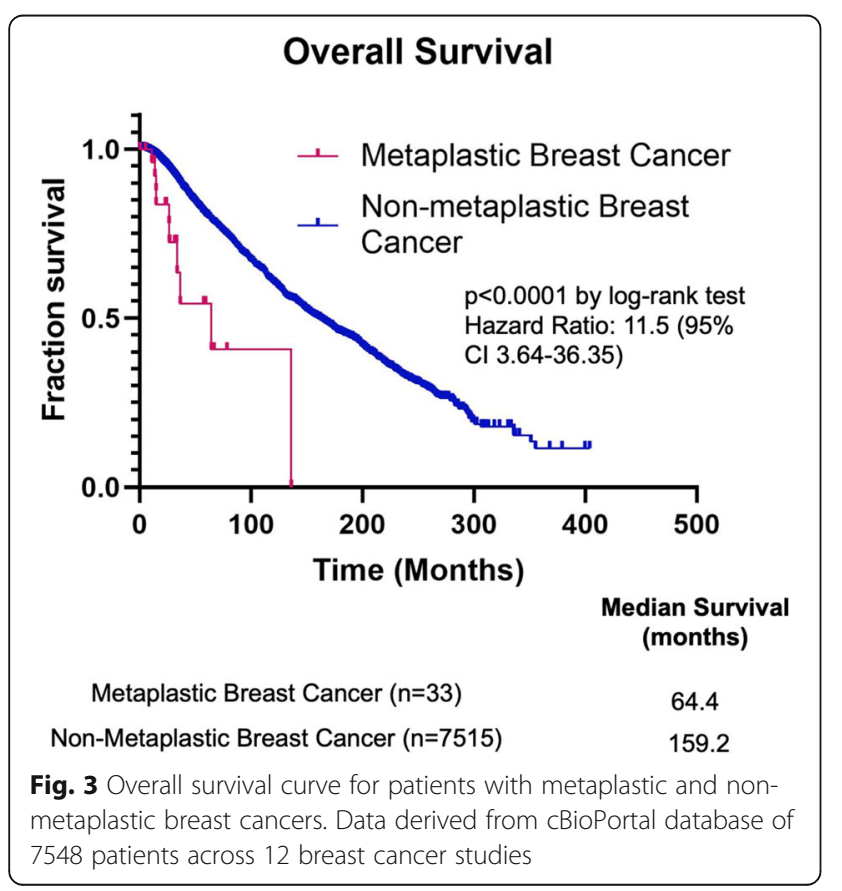

high levels of NO, and the increased iNOS activity may confer resistance to chemotherapy [48]. A preclinical study demonstrated that combining docetaxel with pan-NOS inhibitor NG-monomethyl-L-arginine acetate (L-NMMA) in a xenograft model of MDA-MB-231 decreased Ki67 proliferating cells, enhanced tumor apoptosis, and reduced tumor-initiating capacity of residual tumor cells after chemotherapy to a larger degree than docetaxel alone [29]. Another preclinical study assessed the potential of LNMMA to sensitize metaplastic breast cancer to docetaxel [30]. In this study, L-NMMA significantly reduced cell migration and proliferation in a dose-dependent manner in MpBC cell lines, Hs578T, and BT549. L-NMMA also significantly enhanced docetaxel-mediated apoptosis in $\mathrm{MpBC}$ cell lines, as evidenced by a greater number of Annexin Vpositive cells found in cell lines treated with L-NMMA + docetaxel combination therapy, versus vehicle control or monotherapy. This study also examined tumor samples from patients with histologically confirmed $\mathrm{MpBC}$ and found that out of $40 \mathrm{MpBC}$ samples, 39 samples had a RPL39 A14V mutation. The RPL39 A14V mutation is associated with enhanced NO activity and cancer cell stemness. MpBC tumors that harbored a RPL39 mutation and were resistant to docetaxel became sensitized to the taxane by LNMMA through inhibition of STAT3 signaling. Taking this into consideration, a phase $1 \mathrm{~b} / 2$ clinical trial is being conducted to assess the maximum-tolerated dose, dose-limited toxicities, recommended phase 2 dose, and efficacy of LNMMA in combination with docetaxel for refractory locally advanced and metastatic triple-negative breast cancer patients (NCT02834403). This clinical trial is also recruiting patients diagnosed with $\mathrm{MpBC}$. 


\section{Wnt/ $\beta$-catenin pathway}

The Wnt/ $\beta$-catenin pathway plays a vital role in embryonic development, EMT, and carcinogenesis [32]. In a 2008 study, $36 \mathrm{MpBC}$ samples were analyzed for alterations in the Wnt signaling pathway, by examining immunohistochemical (IHC) stains and mutation analysis of key proteins in the pathway [31]. IHC showed aberrant $\beta$-catenin expression in 33/36 cases (92\%). Mutational analysis demonstrated that $25.9 \%$ of samples had CTNNB1 missense mutations, in the region coding for the NH2-terminal domain of $\beta$-catenin, likely impairing its ability to undergo degradation. APC and WISP3 mutations were seen in $7.4 \%$ and $18.5 \%$ of samples respectively. A subsequent study found a lack of CTNNB1 mutations in $\mathrm{MpBC}$ samples, but more enhanced and focal nuclear localization of $\beta$-catenin in MpBC samples via IHC [32]. A more recent study conducted wholeexome sequencing of $35 \mathrm{MpBC}$ samples and compared the $\mathrm{MpBC}$ genomic landscape to that of TNBC from The Cancer Genome Atlas [27]. The study found that $\mathrm{MpBC}$ more commonly harbored mutations in the Wnt signaling and PI3K/Akt/mTOR pathway than TNBC. These mutations rendered both pathways to be more hyperactive in $\mathrm{MpBC}$. These correlative studies that suggest the Wnt pathway plays a role in $\mathrm{MpBC}$ disease pathogenesis have been translated into preclinical studies, particularly with the development of a mouse model of mammary epithelium-specific Ccn6 protein deletion (MMTV-cre;Ccn6 ${ }^{\mathrm{fl} / \mathrm{fl}}$ ) [33]. CCN6, also known as WISP3, is a matricellular protein involved in development during chondrogenesis, skeletogenesis, and cell attachment to the extracellular matrix. In aggressive cancers such as $\mathrm{MpBC}$, levels of CCN6 are low, which leads to enhanced EMT, invasion, metastasis, insulin-growth factor-1, and bone morphogenic-4 signaling [8]. Studies using the MMTV-cre;Ccn $6^{\mathrm{fl} / \mathrm{fl}}$ model have yielded promising results to suggest that Wnt and IGF-signaling may work synergistically to regulate $\mathrm{MpBC}$ tumorigenesis.

\section{Programmed cell death protein 1 (PD-1)/programmed death ligand-1 (PD-L1)}

A study examined 290 tumor tissues of HR+ and HER2+ breast cancers, $\mathrm{TNBC}$, and $\mathrm{MpBC}$ for immunohistochemical staining of PD-1 on tumor-infiltrating lymphocytes (TILs) and PD-L1 in breast cancer cells [34]. The study found a substantially enhanced expression of PD-L1 on MpBC tumor tissues $(46 \%)$ relative to all other tumor tissues (6\% in HR+ and HER2+, 9\% in TNBC). There was enhanced variability in the expression of PD-1 on TILs in MpBC. Coinciding with this study, a case report published on a patient with metastatic MpBC, from an ongoing clinical trial (NCT02752685), showed a dramatic response to pembrolizumab (anti-PD1) in combination with nab-paclitaxel [35]. PD-L1 staining of the tumor biopsy at baseline showed 100\% of tumor cells were positive for PD-L1, and another staining showed increased TIL infiltration after pembrolizumab treatment. The case report described how the PI3K/Akt and RasMAPK pathways play a role in regulating immune evasion and that the patient's tumor harbored a hyperactivating PIK3CA mutation (H1047R), which may have influenced tumoral PD-L1 expression. Furthermore, EMT, which is a common phenomenon in $\mathrm{MpBC}$, may also influence PDL1 expression. Another study performed PD-L1 immunohistochemical staining of $21 \mathrm{MpBC}$ tumor samples and found that PD-L1 expression was associated with a worse RFS and OS (HR 1.08, 95\% CI 1.01-1.16 and 1.05, 95\% CI $1.00-1.11$, respectively) [28]. Overall, these findings describe the strong potential of immune checkpoint inhibitors in the treatment arsenal for MpBC. Some of the clinical trials described in Table 2 are taking advantage of immunotherapy as a promising therapeutic for $\mathrm{MpBC}$ (NCT02834013, NCT02752685).

\section{Cell cycle regulating proteins}

Krings and Chen conducted a study in which they sequenced 408 cancer-related genes in $28 \mathrm{MpBC}$ samples, and they found that $\mathrm{MpBC}$ harbored a high frequency of TP53 (64\%) and TERT (catalytic subunit of telomerase) promoter mutations (25\%), but the latter frequency varied among $\mathrm{MpBC}$ subtypes [36]. TERT promoter mutations were enriched in the spindle cell and squamous cell carcinoma variants $(47 \%)$. Furthermore, the percentage of TP53 mutations in this study was comparable to another study, which found MpBC to harbor 69\% TP53 mutations [27]. A study examined myoepithelial $\mathrm{MpBC}$ and found that most cases (28 out of $34,82.4 \%$ ) showed a distinct chromosomal loss in 9p21.3, including loss of CDK2NA and $C D K 2 N B$ [37]. $C D K N 2 A$ and $C D K N 2 B$ code for the proteins $\mathrm{p} 16^{\mathrm{INK} 4 \mathrm{a}}$ and $\mathrm{p} 15^{\mathrm{INK} 4 \mathrm{~b}}$, respectively, which function as inhibitors of Cdk4 and Cdk6 and can induce G1 cell cycle arrest by inhibiting the phosphorylation of retinoblastoma protein [49]. Another finding was that loss of 9p21.3 in 64.3\% of all MpBC tumor samples was accompanied by a concurrent PIK3CA mutation.

\section{The role of systemic therapy}

$\mathrm{MpBC}$ is likely to present with more locally advanced disease and a poorer prognosis in comparison to TNBC [50]. The current standard of care for MpBC follows the same guidelines as TNBC, yet $\mathrm{MpBC}$ responds poorly to most systemic chemotherapy and has poorer clinical outcomes than TNBC [50-52]. Chen et al. examined 46 $\mathrm{MpBC}$ cases and found that the partial response rates for $\mathrm{MpBC}$ patients receiving neoadjuvant chemotherapy and first-line chemotherapy were $18.2 \%$ and $8.3 \%$, respectively [50]. None of the patients responded to anthracycline, cyclophosphamide, and vinorelbine-based therapies, but a small cohort of the $\mathrm{MpBC}$ patients 
exhibited a partial response to taxane-based therapy. Aydiner and colleagues conducted an observational study assessing the survival and response to treatment of $54 \mathrm{MpBC}$ and $51 \mathrm{TNBC}$ patients [46]. The study found that $\mathrm{MpBC}$ patients had a decreased response to neoadjuvant chemotherapy (anthracycline and taxane-based therapy) than TNBC patients $(12.5 \%$ vs. $75 \%)$ and that none of the $\mathrm{MpBC}$ patients achieved a complete response to neoadjuvant chemotherapy. In comparison to TNBC, MpBC tends to present with a larger primary tumor size, less nodal involvement, higher histological grade, and heterogeneity, as well as p53 and Ki-67 overexpression [52]. These characteristics may contribute to why $\mathrm{MpBC}$ is more chemorefractory than TNBC.

Despite many studies suggesting that $\mathrm{MpBC}$ is more chemorefractory than TNBC, there is a subset of MpBC patients that may benefit from systemic therapy. Schroeder and colleagues conducted a retrospective study of a MpBC study cohort from the Surveillance, Epidemiology, and End Results Program (SEER) database [53]. The study cohort included 1516 patients diagnosed with $\mathrm{MpBC}$ and 220,375 patients with invasive ductal carcinoma (IDC). Of the MpBC cases examined, $64.1 \%$ were TNBC, 5.2\% were HER2+, and $23.0 \%$ were HER2-/HR+ breast cancers. The 3-year OS for HER2+ MpBC was significantly greater than for triple-negative or $\mathrm{HR}+$ MpBC (91.8\% vs. $75.4 \%$ vs. $77.1 \%, p=0.025)$. Using a multivariate Cox proportional hazards model, the study showed that there was no statistical difference between the OS for HER2+ MpBC cases and HER2+ IDC cases (hazard ratio $=1.16,95 \%$ CI $0.48-2.81, p=.734$ ). Although HER2 + MpBC cases were only a small portion of cases in this study (5.2\%), these findings suggest that systemic therapy, particularly HER2 targeted therapies, may provide improved survival benefit for HER2+ MpBC. Furthermore, this study also suggests that a broader approach to therapeutic options is warranted with $\mathrm{MpBC}$ and that systemic therapies may be efficacious for a particular subset of $\mathrm{MpBC}$ patients.

\section{Radiation therapy}

There have been limited studies and guidelines regarding the use of radiation therapy (RT) in the adjuvant setting for $\mathrm{MpBC}$, and unfortunately, the published studies have patient cohorts that are generally small. Tseng and Martinez conducted a retrospective study in which they investigated a cohort of MpBC patients treated from 1998 to 2006 (SEER database) and found that RT improved the $\mathrm{OS}$ of $\mathrm{MpBC}$ patients following lumpectomy or mastectomy [54]. A case series studying 18 patients with $\mathrm{MpBC}$ showed that patients who underwent postoperative RT had longer overall survival than patients who did not receive RT [55]. A retrospective cohort study by $\mathrm{Li}$ and colleagues in 2019 investigated 2267 patients diagnosed with MpBC between 1998 and 2015 from the SEER database and found that MpBC patients who received RT had a better OS and breast cancer-specific survival compared to those not treated with RT, and this effect was seen particularly in large tumors and elderly patients [56]. However, these studies must be analyzed with caution as either these are retrospective studies, or the studies have a small sample size. To obtain a deeper understanding of how RT can truly benefit MpBC patients in the adjuvant setting, it would be valuable to conduct prospective studies with sufficient sample sizes and develop standardized RT guidelines.

\section{Potential therapies in clinical trials}

Despite the current treatment status for $\mathrm{MpBC}$, which follows a one-size-fits-all scheme in which MpBC patients are provided the same therapeutic options to TNBC patients, there have been completed and ongoing clinical trials specifically on targeted therapeutics against $\mathrm{MpBC}$. For example, a phase 1 clinical trial was conducted to assess the safety and efficacy of mTOR inhibition with temsirolimus or everolimus in combination with VEGF inhibitor bevacizumab and liposomal doxorubicin in 52 patients with advanced MpBC [57]. The objective response rate (ORR) was 21\% (complete

Table $\mathbf{2}$ List of ongoing clinical trials recruiting patients with MpBC

\begin{tabular}{|c|c|c|}
\hline Trial & Phase & Status \\
\hline $\begin{array}{l}\text { ARQule: ARQ751 (pan-AKT inhibitor) plus fulvestrant or paclitaxel compared to ARQ751 plus placebo in patients with breast } \\
\text { or endometrial cancer harboring PIK3CA/AKT/PTEN mutations (NCT02761694) }\end{array}$ & $1 \mathrm{~b}$ & Ongoing \\
\hline $\begin{array}{l}\text { ARTEMIS: A clinical trial implementing diagnostic imaging + tumor genetic signature to predict sensitivity to standard-of-care } \\
\text { versus personalized therapy. A non-randomized trial in which patients undergo baseline imaging and molecular testing of tumor } \\
\text { biopsy. They receive standard anthracycline-based chemotherapy and undergo ultrasound imaging after cycles } 2 \text { and } 4 \text {. After } \\
\text { completing cycle } 4 \text { and obtaining molecular testing results, the patient may elect to continue standard chemotherapy or proceed } \\
\text { to an experimental clinical trial designed to match tumor profile and TNBC subtype. Patients with tumors predicted to be resistant } \\
\text { to standard chemotherapy are advised to participate in the experimental clinical trial (NCT02276443) }\end{array}$ & N/A & Ongoing \\
\hline $\begin{array}{l}\text { L-NMMA (pan-nitric oxide synthase) inhibitor plus docetaxel in refractory locally advanced or metastatic TNBC patients } \\
\text { (NCT02834403) }\end{array}$ & $1 b / 2$ & Ongoing \\
\hline $\begin{array}{l}\text { DART - Dual anti-CTLA4 and anti-PD1 blockade in rare tumors: Nivolumab (anti-CTLA4) antibody) plus ipilimumab (anti-PDL1 } \\
\text { antibody) compared to nivolumab alone for patients with rare tumors, including MpBC (NCT02834013) }\end{array}$ & 2 & Ongoing \\
\hline Pembrolizumab (anti-PD1 antibody) plus nab-paclitaxel for TNBC and HR+/HER2- breast cancer cohorts (NCT02752685) & 2 & Ongoing \\
\hline
\end{tabular}


response $=4[8 \%]$; partial response $=7[13 \%]$, and 10 patients [19\%] had stable disease for at least 6 months, with a clinical benefit rate (CBR) of $40 \%)$. Forty-two tumor samples were available for genetic testing and 32 (74\%) had a PIK3CA mutation. When the presence of a PIK3CA mutation was taken into consideration, they found a significant improvement in ORR (31\% vs. $0 \%)$, but no improvement in CBR ( $44 \%$ vs. $45 \%, p>.99$ ). Though a limitation of this study is the sample size, largely due to the rare nature of $\mathrm{MpBC}$, another point to note is that the PIK3CA mutational analysis was specific to hotspot mutations. There is a chance that the tumor samples of the patient cohort may also harbor other PI3K/Akt pathway alterations that could not be detected via molecular testing. A case report was published in 2019 about a patient diagnosed with MpBC (H1047R PIK3CA mutation +), who enrolled in the BELLE-4 clinical trial, and achieved a durable response and overall survival of 42 months to combination therapy of paclitaxel and buparlisib, a pan-PI3K inhibitor [58]. In addition, immune checkpoint blockade, specifically a combination of nivolumab (anti-PD1) and ipilimumab (anti-CTLA4), has shown efficacy in a small cohort of MpBC patients (The DART trial) [59]. In this prospective, single-arm, phase 2 trial, $17 \mathrm{MpBC}$ patients received combination therapy. The ORR\% in the study was $12 \%$ (RECIST 1.1) and 18\% (iRECIST), with three patients showing ongoing responses at 27,25 , and 23 months, respectively. These smaller studies are first to shed light on the potential of targeting the PI3K pathway and utilizing immunotherapy as treatment options for MpBC. Table 2 provides a list of ongoing clinical trials recruiting patients diagnosed with $\mathrm{MpBC}$.

\section{Conclusion}

Despite the rarity of $\mathrm{MpBC}$, there are well-established molecular drivers of this aggressive chemoresistant subtype, that is, frequently triple negative. In addition to standard treatments, targeting these molecular alterations, either as monotherapy or in combination, is warranted to improve the dismal prognosis of these patients. Innovative strategies targeting PI3K and NOS as well as immunotherapy and radiation should be tested as rational therapies in patients with $\mathrm{MpBC}$.

\section{Supplementary information}

Supplementary information accompanies this paper at https://doi.org/10. 1186/s13058-020-01353-z.

Additional file 1. Supplementary information.

\section{Abbreviations}

MpBC: Metaplastic breast cancer; TNBC: Triple-negative breast cancer; ER: Estrogen receptor; PR: Progesterone receptor; HER2: Human epidermal growth factor 2; PIK3CA: Phosphatidylinositol-4,5-bisphosphate 3-kinase catalytic subunit alpha; TN-IDC: Triple-negative invasive ductal carcinoma; EMT: Epithelial-to-mesenchymal transition; FOXC1: Forkhead Box C1; FOXC2: Forkhead Box C2; Zeb1: Zinc finger E-box-binding homeobox 1; Zeb2: Zinc finger E-box-binding homeobox 2; TIC: Tumor-initiating cell; EGFR: Epidermal growth factor receptor; KIT: Kit proto-oncogene, receptor tyrosine kinase; AKT: Protein kinase B; mTOR: Mammalian target of rapamycin; PIK3R1: Phosphoinositide-3-kinase regulatory subunit 1; TSC2: Tuberous sclerosis complex 2; LKB1: Liver kinase B1; PTEN: Phosphatase and tensin homolog; HR+: Hormone receptor positive; FAT1: Protocadherin fat 1; ARID1A: AT-rich interaction domain 1A; NO: Nitric oxide;

iNOS: Inducible nitric oxide synthase; L-NMMA: NG-monomethyl-L-arginine acetate; RPL39: Ribosomal protein L39; STAT3: Signal transducer and activator of transcription 3; Wnt: Wnt family member 1; CTNBB1: Catenin beta 1;

APC: Adenomatous polyposis coli; WISP3: Wnt-inducible signaling protein 3; CCN6: Cellular communication of network factor 3; MMTV: Mouse model of mammary epithelium; IGF: Insulin-like growth factor; PD-1: Programmed cell death protein 1; PD-L1: Programmed death ligand 1; TP53: Tumor protein 53: TIL: Tumor-infiltrating lymphocytes; MAPK: Mitogen-activated protein kinase; TERT: Catalytic subunit of telomerase; CDK2NA/p16 ${ }^{\text {INK4a }}$ : Cyclin-dependent kinase inhibitor 2A; CDK2NB/P15 $5^{\text {INK4b}}$ : Cyclin-dependent kinase 4 inhibitor B; Cdk: Cyclin-dependent kinase; RT: Radiation therapy; OS: Overall survival; VEGF: Vascular endothelial growth factor; ORR: Overall response rate; CBR: Clinical benefit rate

\section{Acknowledgements}

N/A

\section{Authors' contributions}

All authors were responsible for the idea, preparation, and writing of the manuscript. All authors read and approved the final manuscript.

\section{Funding}

N/A

\section{Availability of data and materials}

The dataset generated and analyzed in the current study are available from cBioPortal, https://www.cbioportal.org/ and information on current clinical trials is from https://clinicaltrials.gov/

\section{Ethics approval and consent to participate \\ N/A}

\section{Consent for publication}

N/A

\section{Competing interests}

The authors declare that they have no competing interests.

\section{Author details}

${ }^{1}$ Houston Methodist Research Institute, 6670 Bertner Ave, Houston, TX 77030, USA. ${ }^{2}$ Texas A\&M Health Science Center College of Medicine, 8447 Riverside Pkwy, Bryan, TX 77807, USA. ${ }^{3}$ Winship Cancer Institute, Emory University School of Medicine, 1365 Clifton Rd, Atlanta, GA 30322, USA. ${ }^{4}$ The University of Texas MD Anderson Cancer Center, 1400 Holcombe Boulevard, Houston, TX 77030, USA. ${ }^{5}$ Houston Methodist Cancer Center/Weill Cornell Medicine, OPC 24, 6445 Main Street, Houston, TX 77030, USA.

Received: 15 April 2020 Accepted: 11 October 2020

Published online: 04 November 2020

\section{References}

1. Lakhani SR El, Schnitt SJ, Tan PH, van de Vijver MJ WHO classification of tumours of the breast, vol. 4, 4th edn. Geneva, Switzerland WHO Press 2012.

2. Howlader N NA, Krapcho M, Miller D, Brest A, Yu M, Ruhl J, Tatalovich Z, Mariotto A, Lewis DR, Chen HS, Feuer EJ, Cronin KA (eds). : SEER Cancer Statistics Review, 1975-2016. In. Bethesda: National Cancer Institute.

3. Weigelt B, Eberle C, Cowell CF, Ng CK, Reis-Filho JS. Metaplastic breast carcinoma: more than a special type. Nat Rev Cancer. 2014;14(3):147-8.

4. Nelson RA, Guye ML, Luu T, Lai LL. Survival outcomes of metaplastic breast cancer patients: results from a US population-based analysis. Ann Surg Oncol. 2015;22(1):24-31. 
5. Huvos AG, Lucas JC Jr, Foote FW Jr. Metaplastic breast carcinoma. Rare form of mammary cancer. N Y State J Med. 1973;73(9):1078-82.

6. Brenner RJ, Turner RR, Schiller V, Arndt RD, Giuliano A. Metaplastic carcinoma of the breast: report of three cases. Cancer. 1998;82(6):1082-7.

7. McCart Reed AE, Kalaw E, Nones K, Bettington M, Lim M, Bennett J, Johnstone K, Kutasovic JR, Saunus JM, Kazakoff S, et al. Phenotypic and molecular dissection of metaplastic breast cancer and the prognostic implications. J Pathol. 2019;247(2):214-27.

8. McMullen ER, Zoumberos NA, Kleer CG. Metaplastic breast carcinoma: update on histopathology and molecular alterations. Arch Pathol Lab Med. 2019;143(12):1492-6.

9. Schwartz TL, Mogal H, Papageorgiou C, Veerapong J, Hsueh EC. Metaplastic breast cancer: histologic characteristics, prognostic factors and systemic treatment strategies. Exp Hematol Oncol. 2013;2(1):31.

10. Tse GM, Tan PH, Putti TC, Lui PC, Chaiwun B, Law BK. Metaplastic carcinoma of the breast: a clinicopathological review. J Clin Pathol. 2006;59(10):1079-83.

11. Oberman HA. Metaplastic carcinoma of the breast. A clinicopathologic study of 29 patients. Am J Surg Pathol. 1987;11(12):918-29.

12. Song Y, Liu X, Zhang G, Song H, Ren Y, He X, Wang Y, Zhang J, Zhang Y, Sun $S$, et al. Unique clinicopathological features of metaplastic breast carcinoma compared with invasive ductal carcinoma and poor prognostic indicators. World J Surg Oncol. 2013;11:129.

13. Downs-Kelly E, Nayeemuddin KM, Albarracin C, Wu Y, Hunt KK, Gilcrease MZ. Matrix-producing carcinoma of the breast: an aggressive subtype of metaplastic carcinoma. Am J Surg Pathol. 2009;33(4):534-41.

14. Koker MM, Kleer CG. p63 expression in breast cancer: a highly sensitive and specific marker of metaplastic carcinoma. Am J Surg Pathol. 2004;28(11): 1506-12.

15. Bataillon G, Fuhrmann L, Girard E, Menet E, Lae M, Capovilla M, Treilleux I, Arnould L, Penault-Llorca F, Rouzier R, et al. High rate of PIK3CA mutations but no TP53 mutations in low-grade adenosquamous carcinoma of the breast. Histopathology. 2018;73(2):273-83.

16. McQuerry JA, Jenkins DF, Yost SE, Zhang Y, Schmolze D, Johnson WE, Yuan $Y$, Bild AH. Pathway activity profiling of growth factor receptor network and stemness pathways differentiates metaplastic breast cancer histological subtypes. BMC Cancer. 2019;19(1):881.

17. Djomehri SI, Gonzalez ME, da Veiga LF, Tekula SR, Chang HY, White MJ, Cimino-Mathews A, Burman B, Basrur V, Argani P, et al. Quantitative proteomic landscape of metaplastic breast carcinoma pathological subtypes and their relationship to triple-negative tumors. Nat Commun. 2020;11(1):1723.

18. Chhieng C, Cranor M, Lesser ME, Rosen PP. Metaplastic carcinoma of the breast with osteocartilaginous heterologous elements. Am J Surg Pathol. 1998;22(2):188-94.

19. Leibl S, Gogg-Kammerer M, Sommersacher A, Denk H, Moinfar F. Metaplastic breast carcinomas: are they of myoepithelial differentiation?: immunohistochemical profile of the sarcomatoid subtype using novel myoepithelial markers. Am J Surg Pathol. 2005;29(3):347-53.

20. Taube JH, Herschkowitz J, Komurov K, Zhou AY, Gupta S, Yang J, Hartwell K, Onder TT, Gupta PB, Evans KW, et al. Core epithelial-to-mesenchymal transition interactome gene-expression signature is associated with claudinlow and metaplastic breast cancer subtypes. Proc Natl Acad Sci U S A. 2010; 107(35):15449-54.

21. Hennessy BT, Gonzalez-Angulo AM, Stemke-Hale K, Gilcrease MZ, Krishnamurthy S, Lee JS, Fridlyand J, Sahin A, Agarwal R, Joy C, et al. Characterization of a naturally occurring breast cancer subset enriched in epithelial-to-mesenchymal transition and stem cell characteristics. Cancer Res. 2009;69(10):4116-24.

22. Creighton CJ, Li X, Landis M, Dixon JM, Neumeister VM, Sjolund A, Rimm DL, Wong H, Rodriguez A, Herschkowitz Jl, et al. Residual breast cancers after conventional therapy display mesenchymal as well as tumor-initiating features. Proc Natl Acad Sci U S A. 2009;106(33):13820-5.

23. Reis-Filho JS, Milanezi F, Carvalho S, Simpson PT, Steele D, Savage K, Lambros MB, Pereira EM, Nesland JM, Lakhani SR, et al. Metaplastic breast carcinomas exhibit EGFR, but not HER2, gene amplification and overexpression: immunohistochemical and chromogenic in situ hybridization analysis. Breast Cancer Res. 2005;7(6):R1028-35.

24. Reis-Filho JS, Pinheiro C, Lambros MB, Milanezi F, Carvalho S, Savage K, Simpson PT, Jones C, Swift S, Mackay A, et al. EGFR amplification and lack of activating mutations in metaplastic breast carcinomas. J Pathol. 2006;209(4):445-53.

25. Gilbert JA, Goetz MP, Reynolds CA, Ingle JN, Giordano KF, Suman VJ, Blair $H E$, Jenkins RB, Lingle WL, Reinholz MM, et al. Molecular analysis of metaplastic breast carcinoma: high EGFR copy number via aneusomy. Mol Cancer Ther. 2008;7(4):944-51.

26. Razavi P, Chang MT, Xu G, Bandlamudi C, Ross DS, Vasan N, Cai Y, Bielski CM, Donoghue MTA, Jonsson $P$ et al: The genomic landscape of endocrineresistant advanced breast cancers. Cancer Cell 2018, 34(3):427-438.e426.

27. Ng CKY, Piscuoglio S, Geyer FC, Burke KA, Pareja F, Eberle CA, Lim RS, Natrajan R, Riaz N, Mariani O, et al. The landscape of somatic genetic alterations in metaplastic breast carcinomas. Clin Cancer Res. 2017;23(14): 3859-70.

28. Afkhami M, Schmolze D, Yost SE, Frankel PH, Dagis A, Amanam IU, Telatar M, Nguyen K, Yu KW, Luu T, et al. Mutation and immune profiling of metaplastic breast cancer: correlation with survival. PLoS One. 2019;14(11): e0224726.

29. Granados-Principal S, Liu Y, Guevara ML, Blanco E, Choi DS, Qian W, Patel T, Rodriguez AA, Cusimano J, Weiss $\mathrm{HL}$, et al. Inhibition of iNOS as a novel effective targeted therapy against triple-negative breast cancer. Breast Cancer Res. 2015;17:25.

30. Dave B, Gonzalez DD, Liu ZB, Li X, Wong H, Granados S, Ezzedine NE, Sieglaff DH, Ensor JE, Miller KD, et al. Role of RPL39 in metaplastic breast cancer. J Natl Cancer Inst. 2017;109(6):1-10.

31. Hayes MJ, Thomas D, Emmons A, Giordano TJ, Kleer CG. Genetic changes of Wnt pathway genes are common events in metaplastic carcinomas of the breast. Clin Cancer Res. 2008;14(13):4038-44.

32. Lacroix-Triki M, Geyer FC, Lambros MB, Savage K, Ellis IO, Lee AH, Reis-Filho JS. Beta-catenin/Wnt signalling pathway in fibromatosis, metaplastic carcinomas and phyllodes tumours of the breast. Mod Pathol. 2010;23(11):1438-48.

33. Martin EE, Huang W, Anwar T, Arellano-Garcia C, Burman B, Guan JL, Gonzalez ME, Kleer CG. MMTV-cre;Ccn6 knockout mice develop tumors recapitulating human metaplastic breast carcinomas. Oncogene. 2017; 36(16):2275-85.

34. Joneja U, Vranic S, Swensen J, Feldman R, Chen W, Kimbrough J, Xiao N, Reddy S, Palazzo J, Gatalica Z. Comprehensive profiling of metaplastic breast carcinomas reveals frequent overexpression of programmed deathligand 1. J Clin Pathol. 2017;70(3):255-9.

35. Adams S. Dramatic response of metaplastic breast cancer to chemoimmunotherapy. NPJ Breast Cancer. 2017;3:8.

36. Krings $\mathrm{G}$, Chen YY. Genomic profiling of metaplastic breast carcinomas reveals genetic heterogeneity and relationship to ductal carcinoma. Mod Pathol. 2018;31(11):1661-74.

37. Bartels S, van Luttikhuizen JL, Christgen M, Magel L, Luft A, Hanzelmann S, Lehmann U, Schlegelberger B, Leo F, Steinemann D, et al. CDKN2A loss and PIK3CA mutation in myoepithelial-like metaplastic breast cancer. J Pathol. 2018;245(3):373-83.

38. Kalluri R, Weinberg RA. The basics of epithelial-mesenchymal transition. J Clin Invest. 2009;119(6):1420-8.

39. Sarrio D, Rodriguez-Pinilla SM, Hardisson D, Cano A, Moreno-Bueno G, Palacios J. Epithelial-mesenchymal transition in breast cancer relates to the basal-like phenotype. Cancer Res. 2008;68(4):989-97.

40. Ribatti D, Tamma R, Annese T. Epithelial-mesenchymal transition in cancer: a historical overview. Transl Oncol. 2020;13(6):100773.

41. Brabletz T, Kalluri R, Nieto MA, Weinberg RA. EMT in cancer. Nat Rev Cancer. 2018;18(2):128-34.

42. Lehmann BD, Bauer JA, Chen X, Sanders ME, Chakravarthy AB, Shyr $Y$, Pietenpol JA. Identification of human triple-negative breast cancer subtypes and preclinical models for selection of targeted therapies. J Clin Invest. 2011;121(7):2750-67.

43. Katso R, Okkenhaug K, Ahmadi K, White S, Timms J, Waterfield MD. Cellular function of phosphoinositide 3-kinases: implications for development, homeostasis, and cancer. Annu Rev Cell Dev Biol. 2001;17:615-75.

44. Janku F, Yap TA, Meric-Bernstam F. Targeting the PI3K pathway in cancer: are we making headway? Nat Rev Clin Oncol. 2018;15(5):273-91.

45. Bae SY, Lee SK, Koo MY, Hur SM, Choi MY, Cho DH, Kim S, Choe JH, Lee JE, $\mathrm{Kim} \mathrm{JH}$, et al. The prognoses of metaplastic breast cancer patients compared to those of triple-negative breast cancer patients. Breast Cancer Res Treat. 2011;126(2):471-8.

46. Aydiner A, Sen F, Tambas M, Ciftci R, Eralp Y, Saip P, Karanlik H, Fayda M, Kucucuk S, Onder $\mathrm{S}$, et al. Metaplastic breast carcinoma versus triplenegative breast cancer: survival and response to treatment. Medicine (Baltimore). 2015;94(52):e2341.

47. Herrera AC, Panis C, Victorino VJ, Campos FC, Colado-Simao AN, Cecchini $A L$, Cecchini R. Molecular subtype is determinant on inflammatory status 
and immunological profile from invasive breast cancer patients. Cancer Immunol Immunother. 2012;61(11):2193-201.

48. Walsh EM, Keane MM, Wink DA, Callagy G, Glynn SA. Review of triple negative breast cancer and the impact of inducible nitric oxide synthase on tumor biology and patient outcomes. Crit Rev Oncog. 2016;21 (5-6):333-51.

49. Ruas M, Peters G. The p16INK4a/CDKN2A tumor suppressor and its relatives. Biochim Biophys Acta. 1998;1378(2):F115-77.

50. Chen IC, Lin CH, Huang CS, Lien HC, Hsu C, Kuo WH, Lu YS, Cheng AL. Lack of efficacy to systemic chemotherapy for treatment of metaplastic carcinoma of the breast in the modern era. Breast Cancer Res Treat. 2011; 130(1):345-51.

51. Invasive Breast Cancer (Version 3.2020) [https://www.nccn.org/patients/ guidelines/content/PDF/breast-invasive-patient.pdf].

52. Jung SY, Kim HY, Nam BH, Min SY, Lee SJ, Park C, Kwon Y, Kim EA, Ko KL, Shin $\mathrm{KH}$, et al. Worse prognosis of metaplastic breast cancer patients than other patients with triple-negative breast cancer. Breast Cancer Res Treat. 2010;120(3):627-37.

53. Schroeder MC, Rastogi P, Geyer CE Jr, Miller LD, Thomas A. Early and locally advanced metaplastic breast cancer: presentation and survival by receptor status in surveillance, epidemiology, and end results (SEER) 2010-2014. Oncologist. 2018;23(4):481-8.

54. Tseng WH, Martinez SR. Metaplastic breast cancer: to radiate or not to radiate? Ann Surg Oncol. 2011;18(1):94-103.

55. Nowara E, Drosik A, Samborska-Plewicka M, Nowara EM, Stanek-Widera A. Metaplastic breast carcinomas - analysis of prognostic factors in a case series. Contemp Oncol (Pozn). 2014;18(2):116-9.

56. Li Y, Chen M, Pardini B, Dragomir MP, Lucci A, Calin GA. The role of radiotherapy in metaplastic breast cancer: a propensity score-matched analysis of the SEER database. J Transl Med. 2019;17(1):318.

57. Basho RK, Gilcrease M, Murthy RK, Helgason T, Karp DD, Meric-Bernstam F, Hess KR, Herbrich SM, Valero V, Albarracin C, et al. Targeting the PI3K/AKT/mTOR pathway for the treatment of mesenchymal triple-negative breast cancer: evidence from a phase 1 trial of mTOR inhibition in combination with liposomal doxorubicin and bevacizumab. JAMA Oncol. 2017;3(4):509-15.

58. Yang MH, Chen IC, Lu YS. PI3K inhibitor provides durable response in metastatic metaplastic carcinoma of the breast: a hidden gem in the BELLE4 study. J Formos Med Assoc. 2019:118(9):1333-8.

59. Adams S, Othus M, Patel SP, Chae YK, Miller K, Chugh R, Schuetze S, Sharon E, Korde LA, Gray RJ, et al. Dual anti-CTLA-4 and anti-PD-1 blockade in metaplastic carcinoma of the breast: Dart (SWOG S1609, Cohort 36). J Clin Oncol. 2020;38(15_suppl):1073.

\section{Publisher's Note}

Springer Nature remains neutral with regard to jurisdictional claims in published maps and institutional affiliations. 